Ann. Biol. anim. Bioch. Biophys., I963, 3 (4), 445-449

\title{
RÉDUCTION EXPERIMENTALE DU NOMBRE DES FCETUS AU COURS DE LA GESTATION DE LA TRUIE ET MAINTIEN DES CORPS JAUNES
}

\author{
F. du MESNIL du BUISSON et P. ROMBAUTS ( $\left.{ }^{1}\right)$ \\ Avec la collaboration technique de A. Chonkiewiecz et A. Locatelti \\ Laboratoire de Physiologie de la Reproduction, \\ Centre national de Recherches zootechniques, Jouy-en-Josas, (Seine-et-Oise)
}

SOMMAIRE

Cinquante-cinq truies nullipares ont été utilisées pour cette étude.

Onze ont subi une hystérectomie subtotale le $\mathbf{i} z^{\mathbf{e}}$ jour de la gestation, en ne sauvegardant qu'un seul foctus et le morceau d'utérus correspondant ; la gestation s'est poursuivie sept fois sur onze.

Les autres ont subi vers le $40^{\mathrm{e}}$ ou vers le $80^{\mathrm{e}}$ jour de la gestation, l'ablation d'un certain nombre de fœetus avec prélèvements du morceau d'utérus correspondant ou sans prélèvement d'utérus. La gestation s'est poursuivie treize fois sur dix-huit dans le premier cas et seulement six fois sur vingt-trois dans le second.

Il est donc possible qu'un morceau d'utérus non gestant ait une action néfaste sur le maintien de la gestation même après la fin du premier tiers de celle-ci; mais nous n'avons jamais trouvé à ce moment d'action unilatérale d'un morceau d'utérus non gravide sur les corps jaunes de l'ovaire correspondant.

Ayant montré précédemment que, chez la 'Truie, l'absence d'embryons dans une corne utérine dès le début de la gestation prévient celle-ci en entraînant la régression des corps jaunes, alors que la présence, à ce même moment, d'un segment de $20 \mathrm{~cm}$ d'utérus non gestant provoque seulement la disparition des corps jaunes du côté correspondant (du MEsNit, du Buisson, I96I), nous nous sommes posés deux questions relatives aux rapports foetus/corps jaune.

I) Une diminution du nombre des foetus au cours de la gestation est-elle susceptible d'interrompre cette dernière?

2) Peut-on faire apparaître par une intervention sur le nombre des foetus, une dyssymétrie lutéale entre les deux ovaires à n'importe quel moment de la gestation?

(1) Une partie de ces expériences ont été faites avec la collaboration de Louis DuUzier, actuellement professeur à I'E........ de Montpellier. Sous l'en remercions très vivement. 


\section{MATÉRIEL ET MÉTHODES}

Cinquante-cinq truies nullipares de 120 à $150 \mathrm{~kg}$ ont été utilisées pour cette étude. onze d'entre elles ont été partiellement hystérectomisées, le $12^{\mathrm{e}}$ jour de la gestation. Les autres truies opérées autour du $40^{\mathrm{e}}$ ou du $80^{\mathrm{e}}$ jour de la gestation ont subi l'ablation d'un certain nombre de fœetus avec prélèvement d'utérus.

Le dosage de la progestérone dans les corps jaunes a été réalisé seulement pour le premier lot, selon une méthode décrite par ailleurs ( $P$. Rombatuts et $C$. Piton, ig63).

\section{RÉSULTATS}

A) Réduction du nombre d'embryons pendant la phase préimplantatoire de la gestation

Onze truies ont été accouplées le deuxième jour de 1'œstrus. Douze jours après l'accouplement, on a procédé à l'ablation de la presque totalité de l'utérus, tout en conservant le cinquième environ d'une des cornes, contigu dans sept cas sur onze au cervix.
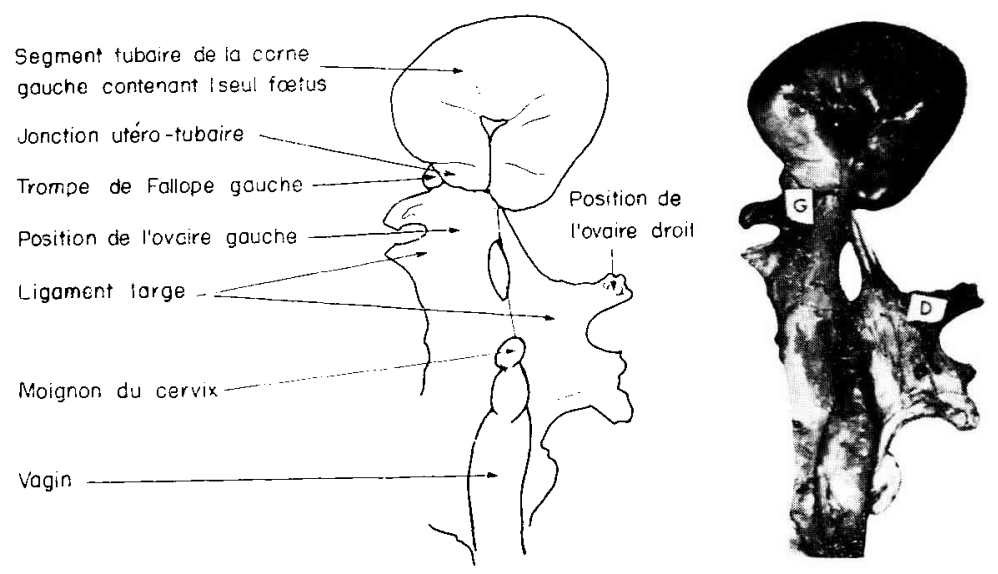

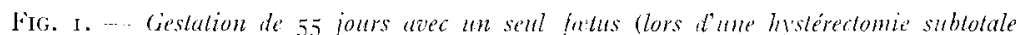
à 12 jours de grestation, seule la portion justa-lubaire de la corne gauche a été conservée)

Six de ces truies ont été abattues 30 jours après l'accouplement et l'on a constaté cinq gestations d'un seul fœutus dans le morceau d'utérus conservé. L'examen des deux ovaires n'a révélé aucune dyssymétrie dans la taille des corps jaunes, dont le nombre n'avait pas varié depuis l'opération. La concentration en progestérone de ces corps jaunes est comparable à celle des corps jaunes de gestation normale :

$67 \mu \mathrm{g}$ par g de tissu lutéal, en moyenne, pour 4 truies à 30 jours de gestation;

$58 \mu \mathrm{g}$ par g de tissu lutéal, pour une truie à 55 jours de gestation.

Une truie est revenue en chaleur 33 jours après l'ostrus préopératoire. Finfin parmi les quatre autres, abattues après 55 jours d'ancestrus, deux étaient gestantes (d'un embryon) et les corps jaunes avaient persisté à droite et à gauche comme dans 
la série précédente; les deux autres étaient vides et l'on a noté la persistance des corps jaunes uniquement du côté opposé à l'utérus comme en cas de réduction utérine durant le cycle (du Mesnil du Buisson, ig6o).

Les résultats négatifs peuvent évidemment être attribués au manque d'embryons dans le segment de corne conservé bien que la réalitè de la gestation ait été vérifiée par perfusion des cornes prélevées au moment de l'opération.

Mais le maintien de sept gestations prouve que les conditions hormonales de la nidation et celles du début de la gestation peuvent être induites par la présence d'un seul embryon à partir du I $2^{\mathrm{e}}$ jour de la gestation; il n'y a pas de relation obligatoire entre le nombre des fœetus et des placenta présents, et celui des corps jaunes pourvu que l'utérus restant soit occupé " en totalité " par le foetus.

\section{B) Réduction du nombre d'embryons dans la phase placentaire de la gestation}

I) Opération faite à la fin du premier tiers de la gestation.

Sur sept truies gestantes de 40 à 43 jours, on a prélevé une corne à l'exclusion des vingt centimètres juxtatubaires. Cette portion d'utérus contenait en général seulement l'extrémité des enveloppes du premier embryon. Dans trois cas, elle a été fendue longitudinalement ; dans quatre cas, elle ne l'a pas été.

Pour les sept truies, la deuxième corne est restée gestante et à l'abattage, après 53 ( $\mathrm{r}$ cas), 60 ( 3 cas) ou 70 jours ( 3 cas) de gestation on a constaté le maintien des corps jaunes, comptés sur chacun des deux ovaires au moment de l'opération. Le poids et l'aspect de ces corps jaunes étaient équivalents des deux côtés.

La réduction du nombre des embryons vers le $40^{\circ}$ jour de gestation n'empêche done pas la poursuite de la gestation au moins jusqu'au $7^{\mathrm{e}}$ jour. D'autre part, la présence d'une portion de $20 \mathrm{~cm}$ d'utérus vide entre 40 et $7^{\circ}$ jours de gestation n'entraîne pas la régression des corps jaunes sur l'ovaire correspondant.

Notons que l'ablation de la totalité des foetus et des cornes qui les contiennent en ne laissant que $20 \mathrm{~cm}$ d'utérus vide juxtatubulaire d'une seule corne, faite au même moment de la gestation ( 3 truies opérées à 42,42 et 53 jours de gestation) permet également le maintien des corps jaunes conme en cas d'ablation de tout l'utérus gestant (du Messir, du Burssox et I)auzier, I959). Cette hystérectomie subtotale n'entraîne pas dans les 30 jours suivant l'opération, la régression des corps jaunes adjacents au segment d'utérus conservé, contrairement à la même hystérectomie subtotale faite sur truie non-gestante pendant la phase lutéale du cycle (du MEsNir. du Buissox, ig6o).

2) Opération faite à la fin du deuxième tiers de la gestation.

Sur onze truies gestantes ( 3 entre 75 et 77 jours de gestation et 8 entre 85 et 95 jours de gestation), on a prélevé l'ensemble des deux cornes utérines en préservant uniquement les deux foetus situés à proximité de la jonction des deux cornes. Six fois sur onze, la gestation s'est poursuivie normalement jusqu'à la mise bas. Dans les autres cas, on a constaté l'avortement 5,5, 10, 20,20 jours après l'opération. 
Les résultats, dans ce cas, sont donc moins bons que lorsque l'opération est effectuée plus tôt, c'est-à-dire vers le $40^{\mathrm{e}}$ jour. On voit cependant que la gestation peut se poursuivre normalement jusqu'à la parturition ( ${ }^{\mathbf{1}}$ ).

\section{C) Excision d'embryon sans prélèvement d'utérus}

L'ablation d'un ou de plusieurs fœetus faite sans décoller le placenta et sans prélever le morceau d'utérus correspondant a entraîné très souvent l'avortement dans la semaine suivant l'intervention. Cette réaction a été génétale pour huit truies opérées entre 68 et 89 jours de gestation.

Et sur quinze truies opérées à 40 jours de gestation, on a constaté seulement six grossesses de 70 ou 90 jours; au moment de l'abattage, le segment d'utérus correspondant à l'emplacement des embryons prélevés se trouvait " non gestant », car les enveloppes restantes étaient décollées, exangues et nécrosées. Les corps jaunes étaient équivalents sur les deux ovaires.

Les avortements consécutifs à l'ablation du foetus seul peuvent être attribués, en même temps qu'à l'infection ou au traumatisme, à une action néfaste de la portion de corne non gravide.

En conclusion, l'ablation d'une partie des fœtus avec le segment de corne utérine qui les contient, permet la plupart du temps le maintien de la gestation, (7/ro, 7/7 $6 /$ Ir) alors que l'ablation de foetus seuls semble être un facteur d'avortement (maintien de la gestation, $6 / 15$ et $o / 8$ ).

Mais en cas de gestation la présence d'un segment d'utérus non gestant à partir de la fin du premier tiers de celle-ci ne provoque aucune régression dyssymétrique des corps jaunes entre les deux ovaires.

L'action "locale anti-corps jaune " de l'utérus non gravide est donc liée à la période de transformation du corps jaune cyclique (préimplantation) en corps jaune de gestation proprement dit, soit par suite d'une sécrétion spécifique de l'utérus, soit par une particulière sensibilité du corps jaune à ce moment.

\section{Reçu pour publication en décembre 1963.}

\section{SUMMARY}

EXPERIMENTAL REDLCTION OF THE NUMBER OF EMBRYOS 1)URING GESTATION OF TIE GILT AND MAINTENANCE OF THE CORPORA LUTEA

Fifty-five gilts were used in these experiments.

Eleven animals were subtotally hysterectomized on the 12 th day of gestation. All of the uterus was removed, except one embryo and its corresponding portion of horn. Gestation continued in 7 of these gilts.

In $4 \mathrm{I}$ other gilts, removal of a certain number of embryos with or without the corresponding portions of the uterine horn was performed around the 4 oth or 8oth day of gestation. Gestation continued in 12 of 18 animals in which some embryos with their corresponding uterine horns were removed, whereas only 6 of 23 animals remained pregnant when only embryos were removed.

(I) $G_{0}$ ćtant le jour de l'accouplement, les mise bas ont eu lieu à $G_{115}$. $G_{115}, G_{114}, G_{117}$, deux truies ont étés abattues à $\mathrm{G}_{115}$, et $\mathrm{G}_{113}$; ces chiffres sont supérieurs à la durée moyenne de la gestation des truies Large While. 
It is possible that a portion of empty uterine horn has a negative influence on the maintenance of pregnancy after the first 40 days of the gestation. Howewer, uterine luteolytic activity of the empty uterine horn was not apparent on the corresponding ovary after the 4 oth day of gestation.

\section{RÉFÉRENCES BIBLIOGRAPHIQUES}

du Mesnil du Buisson F., т960. Régression unilatérale des corps jaunes aprís hystérectomie partielle chez la 'Truie. Ann. Biol. anim. Bioch. Biophys., 1, 105-112.

du Mesnil du Busssox F., rg6r. Possibilités d'un fonctionnement dissemblable des ovaires pendant la gestation de la Truie. C. R. Acad. Sci., 253, 727-729.

du Mesnil du Buisson F. et Daczier L., J959. Contrôle mutuel de l'utérus et de l'ovaire chez la Truie. Ann. Zoolech., supp., I 47-159.

Rombatts P. et Pitox C., 1963. Amćlioration du dosaga de la progestérone sous forme de dinitrophénylhydrazone Inn. Biol. anim. Bioch. Biophys., 3, $437-4+3$. 